\title{
Théologiques
}

Théologiques

\section{Le charisme}

\section{Aux frontières de la sociologie}

\section{Johannes Weiss}

Volume 17, numéro 1, 2009

Les charismes

URI : https://id.erudit.org/iderudit/039496ar

DOI : https://doi.org/10.7202/039496ar

Aller au sommaire du numéro

\section{Éditeur(s)}

Faculté de théologie et de sciences des religions, Université de Montréal

ISSN

1188-7109 (imprimé)

1492-1413 (numérique)

Découvrir la revue

Citer cet article

Weiss, J. (2009). Le charisme : aux frontières de la sociologie. Théologiques, 17(1), 15-32. https://doi.org/10.7202/039496ar
Résumé de l'article

Le présent essai cherche à observer et à comprendre pourquoi la sociologie s'accommode si difficilement des exceptions dont la portée est générale, exceptions qui se caractérisent par une "distinction ou [... un] talent spécial ". Pour ce faire, il retrace d'abord le traitement du charisme dans la discipline sociologique à partir de la réception des écrits de Max Weber sur ce concept. Il plaide ensuite pour un retour à la position originale de Weber, pour qui le charisme ne saurait être réduit à une " catégorie résiduelle ", mais représente bien plutôt un concept limitrophe et fécond de la sociologie.
Ce document est protégé par la loi sur le droit d'auteur. L'utilisation des services d'Érudit (y compris la reproduction) est assujettie à sa politique d'utilisation que vous pouvez consulter en ligne.

https://apropos.erudit.org/fr/usagers/politique-dutilisation/ 
Théologiques 17, no 1 (2009) p. 15-32

\title{
Le charisme
}

\section{Aux frontières de la sociologie ${ }^{1}$}

\author{
Johannes WeISS* \\ Professeur émérite \\ Université de Kassel, Allemagne
}

1.

"Mais il y a les exceptions. Si l'on ne peut les expliquer, on ne peut davantage rendre compte du général» (Kierkegaard 1972, 93). Cette phrase de Kierkegaard, issue de La répétition, concerne d'abord «le caractère extraordinaire » propre à de grands personnages, tels les "prophètes et apôtres " de l'histoire de la révélation. Sans la perception et la compréhension de ce que de tels êtres exceptionnels ont pu accomplir, on ne saurait rendre compte de l'histoire de la révélation et de la croyance en sa totalité.

Ce fondement kierkegaardien ne concerne pas seulement ces quelques personnages, il renvoie également à l'exceptionnel et à l'extraordinaire propres à tous les hommes en tant qu'individus uniques, à ce qui correspond, selon le philosophe, à la relation religieuse: Dieu "entend », dit-il, "grâce aux individus, c'est-à-dire grâce à chacun d'eux, en faire sortir le bien suprême» (Kierkegaard 1979, 227). Pour cette raison, «il faut tout d'abord prendre soin que tout homme soit un Individu et en ait conscience » (Kierkegaard 1971, 271). L'aspect désolant dans l'Antiquité fut justement "que l'esprit supérieur était ce que les autres ne pouvaient être » alors que

1. Ce texte a été traduit par Barbara Thériault et Louis-Philippe Vien.

* Johannes Weiss est professeur émérite à l'Université de Kassel. Ses domaines de recherches incluent la théorie sociologique, la philosophie des sciences sociales et la sociologie de la culture. Ces livres sont notamment parus sous sa plume: Weber and the Marxist World (Londres 1986, nouvelle édition 1998), Vernunft und Vernichtung. Zur Philosophie und Soziologie der Moderne (Opladen 1993), Handeln und handeln lassen. Über Stellvertretung (Opladen 1998) ainsi que le volume collectif Die Jemeinigkeit des Mitseins. Die Daseinsanalytik Martin Heideggers und die Kritik der soziologischen Vernunft (Konstanz 2001).

(C) Revue Théologiques 2009. Tout droit réservé. 
"quiconque s'est conquis lui-même au plan religieux est simplement ce que tous peuvent être» (Kierkegaard 1979, 212).

Cette phrase de Kierkegaard concernant l'exceptionnel et le général aborde donc le caractère individuel dans un sens double: «au sens de la distinction ou du talent spécial ", mais aussi "au sens où tout homme sans exception peut et doit l'être» (Kierkegaard 1971, 93).

\section{2.}

Tout cela, Kierkegaard l'opposait d'abord et avant tout à la théologie et à l'Église chrétienne (qui se voulait chrétienne) de son temps. En cela repose un défi fondamental pour toutes les sciences de l'homme, et en particulier pour la sociologie ${ }^{2}$. En effet, l'idée fondatrice et la raison d'être de cette science reposent sur l'hypothèse que l'existence humaine est de nature essentiellement sociale, qu'elle est rendue possible, déterminée et délimitée par le social. C'est pourquoi l'exceptionnel, voire le singulier, est pour la sociologie soit peu pertinent ou négligeable, soit perçu comme une fiction dont l'émergence et la fonction ne se laissent expliquer que par le recours à des règles et à des conditions sociales.

L'essentiel de ce qui sera abordé dans cet essai se résumera à observer et à comprendre pourquoi la sociologie s'accommode si difficilement des exceptions dont la portée est générale, exceptions qui se caractérisent par une «distinction ou [... un] talent spécial». Il est au demeurant dans la logique de la chose, et non seulement dans la position de Kierkegaard, que les réflexions se détourneront du particulier dans son sens extraquotidien vers ses formes d'existence proprement quotidiennes et, ainsi, vers des questions élémentaires de la connaissance sociologique.

2. Il serait grand temps d'accorder à Kierkegaard l'attention qui lui revient du point de vue sociologique, et ce, comme quelqu'un qui fut, à l'instar de Friedrich Nietzsche, un sociologue avant la lettre et un critique de la sociologie. Il serait important de réfléchir, dans le cadre des controverses actuelles sur l' "individualisation », à ce que Kierkegaard qui, anticipant Nietzsche, avait à dire à propos «des temps modernes" et de «l'abstraction désolante du nivellement» qui les caractérise. «Le principe d'association (tout au plus recevable dans le domaine des intérêts matériels) est de nos jours négatif et non affirmatif; il est prétexte, distraction, chimère, et sa dialectique est la suivante: en réconfortant les individus, il les énerve: il leur rapporte le réconfort du nombre, de la solidarité, mais pour l'éthique c'est là un affaiblissement ». (Kierkegaard 1979, 224) 
3.

Au-delà des questions d'interprétation, on pourra objecter que Kierkegaard n'est pas un sociologue; et on pourra invoquer que l'un des plus importants sociologues à ce jour, un "classique " comme Max Weber, a fait de l'existence et de l'impact des personnages exceptionnels un thème central de son œuvre. En effet, c'est lui qui a introduit en sociologie le concept de domination charismatique comme l'une des trois formes idéaltypiques de domination légitime et qui a démontré le caractère incontournable et fécond du concept de charisme dans ses enquêtes empiriques, particulièrement dans le champ de la sociologie comparée des religions.

S'il serait aussi faux de prétendre que la notion de charisme fut omise ou méconnue par les sociologues qui ont succédé à Weber, comme ce fut le cas d'autres éléments fondamentaux de son œuvre (surtout en ce qui a trait à sa méthodologie), la problématique du charisme a toutefois toujours éveillé les soupçons. Conjuguée au principe individualisant qui serait à la base de sa théorie et de sa méthodologie, l'insistance de Weber sur la problématique du charisme et son traitement a fait naître l'idée que sa pensée était chargée au plan idéologique, qu'elle était bourgeoise et élitaire, donc antisociale et, dans cette mesure, anti-sociologique. Une telle critique peut s'appuyer sur le fait, incontestable et souligné par Weber lui-même, que sa pensée fut fortement influencée par celle de Nietzsche. Ce dernier n'avaitil pas lui-même fustigé cette nouvelle science - du moins celle représentée par Herbert Spencer et John Stuart Mill ? Ne critiquait-il pas cette nouvelle science pour faire de la "société " et, avec elle, de la relativité (Vermittelmässigung) de l'homme et des relations humaines, non seulement son objet, mais aussi la mesure de toutes choses? Cette science ne déclaraitelle pas ainsi la guerre à tous les ordres hiérarchiques, à toutes les distinctions et à toute individualité forte? Et Nietzsche n'avait-il pas été à cet égard fondamentalement inspiré par Jacob Burckhardt dont l'enseignement sur le rôle historique des "grands individus» s'était alimenté de motifs aristocratiques, et donc politiquement réactionnaires ${ }^{3}$ ?

3. Cette question ne sera pas approfondie ici. La perception manifestement très affirmative de Nietzsche du prophétisme doit aussi être laissée de côté, et cela même si celle-ci, particulièrement l'idée du lien du prophète à son grand «œuvre », inspira Weber (voir 2005, 45). 
4.

Bien que ce ne soit pas étonnant, il convient d'évoquer que c'est particulièrement la critique marxiste de Weber qui a emprunté cette voie. Au sein du marxisme et par-delà ses frontières, La destruction de la raison de Georg Lukács (2006 [1954]) a longtemps donné le ton et la direction de cette critique, et ce, même si Theodor W. Adorno (qui se réclamait lui-même de cette tradition théorique) a fait remarquer que ce livre n'instruisait que sur la destruction de la raison de son auteur.

Le fondement individualiste, donc bourgeois, anti-égalitaire et antidémocratique de cette tradition intellectuelle spécifiquement allemande fondement qui était ainsi, par ricochet, antisocial et pour cette raison aussi irrationnel —, remonte d'abord et avant tout à Nietzsche. Elle trouve son expression la plus forte et la plus influente dans la sociologie de Max Weber ainsi que dans celle de Georg Simmel.

En raison de la conception du charisme en général et de l'idée connexe d'une "démocratie plébiscitaire des chefs" (en particulier au regard du nouvel ordre politique de l'Allemagne après la Première Guerre mondiale), Max Weber appartient, selon Lukács et plusieurs autres auteurs, à ceux qui ont préparé le terrain au fascisme, du moins sur le plan des idées ${ }^{4}$.

Pendant longtemps, il n'y avait pas que les cercles marxistes pour critiquer le concept wébérien du charisme. De façon certes moins virulente, on ne l'évoquait qu'avec une prudence manifeste. Si cette méfiance était en partie motivée d'un point de vue politique, elle émergea essentiellement de l'opinion mentionnée précédemment qui veut que Weber se soit aventuré à l'extrême limite ou à l'extérieur de ce qui était pertinent et concevable en sociologie. C'est ainsi que même un "wébérien » aussi convaincu et productif que Dirk Kaesler avança que la domination charismatique constituait une "catégorie résiduelle» (cité par Hatscher 2000, 19). Par là, il faisait référence à un concept sous lequel Weber aurait subsumé des phénomènes qui ne pouvaient pas être définis de façon précise, mais qui ne devaient pas non plus être laissés-pour-compte.

4. Pour une critique marxiste de Weber, voir la postface de La destruction de la raison (Lukács 2006), ainsi que Weiss (1998a). 
5.

Depuis quelque temps, ce traitement critique, sceptique, ou pour le moins réservé de la sociologie wébérienne du charisme a fait place à une discussion productive qui ne cesse de s'intensifier et de croître ${ }^{5}$.

Certes, on affirme avec raison que la dynamique de la modernité a entretemps été rompue et, de façon concomitante, que le champ d'émergence et d'effet des personnalités charismatiques est disparu jusqu'à nouvel ordre ${ }^{6}$. En effet, dans sa forme originelle et toute puissante, le charisme semble être devenu quelque chose de suranné, son "âge d'or " relégué au passé. L'usage actuel des mots «charisme » et «charismatique» dans le langage de la politique, de la publicité, de la vie de tous les jours ainsi que dans l'industrie du spectacle témoigne de l'inflation de sens et de valeur qui le caractérise aujourd'hui.

Il est peu probable que le charisme puisse, à l'heure actuelle, engendrer la force d'impulsion d'un renouveau qui ébranlerait les fondements culturels ou politiques ou qui engendrerait une réorientation radicale, une metanoia. Le charisme, dans sa forme "originelle», ne peut pas être inventé, fabriqué au besoin ou appris par des gens talentueux. Si le charisme authentique au sens que lui confère Weber ne peut pas être généré ou enseigné ad libitum, il peut être éveillé et encouragé dans son déploiement.

Weber lui-même avait tendance à voir ici une sérieuse - mais inévitable - carence de la culture moderne, rationnelle et sans prophète. Il critiquait d'autant plus sévèrement les prétentions charismatiques et les mises en scène du charisme auxquelles s'adonnaient de nombreux intellectuels de son entourage. Il rejetait catégoriquement ce qu'il qualifiait de romantisme intellectualiste moderne de l'irrationnel (Weber 2005, 35). Le danger d'une régression intellectuelle ou morale, d'une dépréciation et d'une dilution des standards reçus du savoir et de la moralité, accompagne comme une ombre les rapports de domination sous-tendus par le charisme. Cela dit, faire de cette régression une condition préalable aux rapports de domination charismatique renverrait à une inversion complète des choses ${ }^{7}$.

5. Voir l'aperçu et la bibliographie de la littérature récente qui l'accompagne dans l'introduction de Gebhardt (1993).

6. À cet effet, voir Rieff (2008). Toutefois, celui-ci affirme que le rôle de Max Weber a été de dissocier l'idée du charisme de son rapport authentiquement religieux (chrétien), de la réinterpréter d'un point de vue politique et, ainsi, de la neutraliser.

7. Dans ce contexte, la manière dont Weber se serait exprimé quant au grand succès que connut la prétention charismatique d'Hitler (à l'encontre non seulement de ce qu'ont longtemps prétendu non seulement des idiots, mais aussi d'excellents savants — les philosophes n'étaient en reste) est claire. 
6.

L'absence du charisme originel dans le présent et son caractère peu plausible dans un futur proche ne le rendent d'aucune façon inintéressant pour la sociologie. L'expérience de la perte représente ici un grand défi, du moins dans la mesure ou l'on ne s'accommode pas d'un concept de charisme qui se laisserait explicitement extrapoler au-delà de la conception wébérienne, ou que l'on ne considérerait que dans son état de dépersonnalisation, d'objectivation et d'institutionnalisation pour ainsi trouver la trace de l'extraquotidien dans le quotidien.

Gebhardt (1993; 1994, 24 sq.) a noté que la recherche sur le charisme qui se réclame de Weber a, durant les deux dernières décennies, précisément exécuté cette volte-face. On critique ainsi les enquêtes antérieures surtout celles portant sur la sphère politique — pour avoir trop insisté sur les personnalités charismatiques. On argumente que le potentiel de connaissance qu'offre l'approche wébérienne peut davantage être exploité si l'on se libère de cette simplification et redirige l'attention vers les aspects du charisme qui se manifestent en dehors et indépendamment des êtres exceptionnels.

Il est certes légitime et fécond d'orienter la recherche dans cette direction (comme l'ont notamment fait Roth [1987] et Schluchter [1991] dans leurs travaux). Malgré tout, on ne devrait pas oublier que, pour Weber, le charisme est dans sa forme primaire et intrinsèque toujours le charisme d'une personne dotée de capacités particulières en vertu desquelles elle est appelée à diriger et à bouleverser des rapports existants. Stefan Breuer, dont l'enquête sur le "charisme de la raison» (1993) est subsumée par Gebhardt sous ce nouveau courant de recherche sur le charisme, parle pour cette raison, tout comme Weber, du charisme "originel». Il serait toutefois important de vérifier si l'on doit à cet effet comprendre le "charisme de la raison " comme le dernier degré de la "dépersonnalisation et de l'objectivation du charisme» comme l'affirme Breuer (1993, 181). Peut-on vraiment rendre compte de la dynamique révolutionnaire du «jacobinisme» en faisant abstraction des individus considérés comme charismatiques tels Robespierre ou Saint-Just, «le saint Jean du Messie du peuple» (Lamartine, 1847) ? L'imposition révolutionnaire du léninisme peut-elle être expliquée convenablement sans son éponyme et ses protagonistes, comme Breuer le croit manifestement? Doit-on imputer au «charisme de la raison» ou encore au «charisme de la science» (Weber 1972, 657) des formes dépersonnalisées et objectivées du charisme, le succès, du moins momentané, 
des grands mouvements révolutionnaires inspirés du marxisme? Ou doiton l'imputer à son incarnation et à sa personnification dans des individus extraordinaires (ou tenus pour hors du commun) tels Lénine, Trotski et aussi Staline, Mao, Hô Chi Minh et Castro? Ces personnages ne doivent-ils pas être considérés comme conditions fondamentales et irremplaçables du succès de ces mouvements?

\section{7.}

C'est ce charisme " originel », personnel, ainsi que la place particulière que Weber lui confère qui ont été, selon Breuer (1993), une source constante d'agacements et de critiques. Le détournement du charisme de son sens «originel » proposé, louangé et pratiqué par Gebhardt peut s'expliquer, si on garde en tête les considérations introductives de ce texte, par l'intérêt à déterminer des objets de recherche et à formuler des questions de recherche, lesquels ne s'exposent pas à un tel degré d'agacement puisqu'ils sont véritablement sociologiques, bien qu'ils ne soient pas véritablement wébériens. Il s'agit donc, pour le dire rapidement, d'observer la problématique du charisme à partir de la perspective qui s'insère de façon «irréprochable » dans le modèle standard de la taxinomie et de la théorie sociologiques. Au lieu de se laisser agacer par cette problématique, la sociologie se l'approprie (l'objet en soi ainsi que l'appareil conceptuel, théorique et méthodologique qui permet de le saisir) selon ses (prétendus) besoins et ses possibilités.

Le défi et le gain théorique possible reposent cependant sur la manière précise dont Max Weber a délibérément introduit le charisme dans son "système » sociologique, c'est-à-dire pour désigner la qualité propre d'acteurs sociaux singuliers, ou du moins considérés comme singuliers. Pour cette raison, il convient de se questionner sur la signification et les implications de cette conceptualisation. Il s'agit de la tâche qui nous incombe ici, et ce, dans une double perspective. Une première question concerne la façon dont la domination charismatique se présente lorsqu'on l'appréhende en tant que "relation sociale", au sens de la sociologie compréhensive. La réponse à cette première question, en soulève une deuxième: de quelle façon et dans quelle mesure le sens visé par les acteurs d'une telle relation sociale se laisse-t-il appréhender par la «compréhension par interprétation» de la sociologie? Et quelles limites la méthodologie impose-t-elle à cette compréhension? 


\section{8.}

Le charisme est, dans la perspective wébérienne, d'abord et avant tout une distinction propre à des personnes déterminées qui les rend aptes à la domination et qui légitime cette dernière. Ce type de domination n'est du reste absolument pas limité à la sphère politique ${ }^{8}$. Selon Weber, il convient de parler de domination lorsque «des ordres spécifiques» peuvent «trouver obéissance de la part d'un groupe déterminé d'individus» (1995, 285).

Ainsi comprise, la domination ne pourrait se perpétuer sans une «volonté d'obéissance» de la part des dominés. Cette volonté témoigne de façon exemplaire de la reconnaissance du charisme «strictement personnel » et de la "dévotion" au chef qui en résulte. Plusieurs motifs peuvent toutefois sous-tendre cette volonté d'obéissance. Comme presque partout ailleurs, on pourra détecter, dans le cas d'une domination légitimée principalement de façon charismatique, des motifs purement rationnels ou utilitaires. Mais ce ne seront ici ni des motifs utilitaristes ni des motifs traditionnels, mais bien des motifs (selon la typologie wébérienne) affectuels, ou rationnels en valeur, qui seront déterminants pour évaluer la chance d'un ordre de trouver obéissance (la disposition à l'assujettissement et à la pérennisation de cet état). De façon idéaltypique, la domination charismatique s'avérera la plus achevée (et cela signifie aussi que sa chance d'avoir un impact sur la réalité sera plus grande) lorsque la relation affective inconditionnelle à une personne se conjugue avec la croyance rationnelle en valeur que cette personne incarne la vérité absolue de certaines interprétations du monde, ou la justesse absolue d'impératifs religieux, politiques ou moraux qu'elle est destinée à accomplir.

Par opposition à une relation hautement personnelle et affective sous le signe de l'amitié ou de l'amour, une relation de domination motivée par le charisme se caractérise par la création de quelque chose de nouveau et de grand, quelque chose qui, d'abord à l'intérieur puis à l'extérieur, refonde le monde de fond en comble. Les qualités extraordinaires qui caractérisent les dominants et qui motivent, ou devraient motiver, les dominés, doivent donc

8. On peut conférer, selon la nature de la relation sociale, plusieurs sens au terme wébérien de «domination» (Herrschaft). Généralement, ce terme signifie «direction », ou un rapport guide-adeptes. Les dominants, particulièrement ceux qui tirent leur légitimité d'un charisme, peuvent aussi être perçus et agir en tant que "modèles " (idéaux, donc inaccessibles), en tant que héros moraux par exemple. Ce sens nous rappelle la dichotomie précisée par Weber dans "L'introduction» à sa sociologie comparée des religions, celle qui distingue prophétie «exemplaire» et prophétie de "mission » (1996, 355). 
se référer à ce grand œuvre, à son invention, sa détermination et sa réalisation. Une telle domination ne se maintient que dans la mesure où elle est confirmée. Le caractère exceptionnel des dominants doit toujours être confirmé à nouveau: d'une part par la force de persuasion que sous-tend et incarne cet œuvre par son urgence, et d'autre part par la preuve qu'elle peut se réaliser et s'accomplir sous leur domination.

Sous cet angle, la soumission à des personnes perçues comme charismatiques n'est pas inconditionnelle: la confiance des dominés n'est pas aveugle et leur disposition à suivre un chef n'est pas, de leur point de vue, «irrationnelle». Les dominés savent ou (ce qui revient au même dans les faits) croient savoir ce qu'ils font et pourquoi ils le font. Ils n'agissent pas de façon irréfléchie, dans l'enivrement, du moins au-delà de la phase initiale. Cette phase est toutefois déterminée par une forte expérience de la "nécessité et de l'enthousiasme " (Weber 1995, 325) d'un émoi né de l'extraordinaire et communément expérimenté par un groupe de personnes. Il s'agit de l'attente de l'arrivée de quelque chose de prodigieusement grand et salutaire. Et cette attente doit connaître récompense. La non-réalisation de son objet fait disparaître le charisme, et avec lui la croyance en la légitimité et la volonté de s'y soumettre.

\section{9.}

Dans ses écrits méthodologiques, Weber mentionne occasionnellement que l'action, dans la mesure où elle est dotée d'un sens et que les motifs qui la sous-tendent sont «communicables» entre les sujets, possède une rationalité «qualitative». Il n'a cependant jamais introduit dans sa terminologie ni explicité ce qu'il entendait par «communicable» et «qualitativement rationnel ». Il ne l'a jamais développé de façon systématique, et ce, même lorsqu'il entreprit enfin de mettre sur papier le «système» de la sociologie compréhensive et, dans cette foulée, de préciser le sens de l'activité sociale à l'aide du concept de la communicabilité (voir à cet effet Weiss 1993, 216 sq.; $1998 \mathrm{a}, 133$ sq. et 155 sq.). Il n'y a pourtant aucun doute qu'il tenait fermement à une telle perspective comme en témoigne le déplacement du concept fondamental de valeur vers celui de sens.

C'est sous cet angle que l'on doit considérer la domination charismatique. Cette perspective implique une question: qu'en est-il de la rationalité qualitative en tant que communicabilité ? Il s'avère que ce type de domination ne pose pas de problème particulier ou fondamental à la compréhension par interprétation et à l'explication causale qu'elle sous-tend. Comme 
nous le rappelle Weber, «il n'est pas besoin d'être César pour comprendre César» $(1995,29)$ et l'on n'a pas besoin non plus d'être un Romain de l'époque, de classe et d'éducation spécifiques pour comprendre, au niveau du sens, pourquoi l'on pouvait succomber à la force extraordinaire d'attraction et de persuasion de cet homme (de ce héros guerrier) ou pourquoi, justement pour cette raison, on pouvait croire qu'il fallait le faire disparaître. Les capacités extraordinaires, en raison desquelles les uns suivirent César et les autres attentèrent à sa vie, sont en principe compréhensibles pour les historiens et les sociologues d'aujourd'hui, et non seulement pour les hommes qui étaient contemporains de César. Une telle compréhension, naturellement hypothétique, peut et doit, dans une forme contrôlée du point de vue de la méthode, préfigurer toute explication historique ou historico-sociologique. À cet effet, le contenu particulièrement chargé de sens (Sinn-Gestalt) de la condition charismatique lui est bienvenu. Il va sans dire que la recherche scientifique est ici tenue d'opérer avec une extrême prudence méthodologique. En effet, l'hypothèse du charisme possède un caractère d'évidence qui tend à s'imposer facilement comme une explication causale qui ne nécessiterait aucune vérification supplémentaire. La domination charismatique pure, observe Weber, se présente souvent comme composante d'une formation historique et empirique construite purement et seulement aux fins de l'observation théorique $(1995,326)$.

\section{0.}

Cela dit, l'affirmation voulant que la domination charismatique paraisse et demeure irrationnelle d'un point de vue sociologique est assez convaincante. En effet, le charisme s'appréhende suivant une définition qui témoigne d'une chose qui est non seulement extraquotidienne, mais qui passe également pour absolument surnaturelle, et donc comme un objet auquel la rationalité scientifique ne peut prétendre puisqu'il ne peut être ni démontré ni falsifié. Mais ne s'agit-il pas en fait d'un cas similaire à l'étude de complexes d'actions ou de motifs dont le sens subjectivement visé ne se laisse pas traduire dans le langage de la sociologie (ni dans celui de la psychanalyse ou de l'économie) sans rendre leur signification causale incompréhensible? La connaissance sociologique ne vise pas la réalité ou la vérité du sens visé, mais plutôt les motifs qui peuvent, voire doivent, conduire des hommes à croire dans certaines circonstances en la réalité et la vérité de forces surnaturelles, impalpables et non vérifiables d'un point de vue empirique. Ce type de connaissance est possible lorsqu'on inclut d'une part la nécessité et 
le devoir qui transcendent toute mesure normale, et dont le traitement nécessite des forces surhumaines ou surnaturelles, et lorsqu'on doit présumer d'autre part que les hommes et les femmes concernés ne s'en tiennent (ou ne peuvent s'en tenir) qu'à la perception et à l'attribution de telles forces et capacités situées aux limites de la vérification empirico-scientifique.

\section{1.}

On pourrait bien sûr être d'accord avec une telle position, mais néanmoins insister sur le fait que la sociologie atteint ici une limite définitive. Lorsqu'il s'agit des formes d'attributions charismatiques, la sociologie s'en tiendra, du moins semble-t-il, à une indifférence stricte, comme si le charisme devait toujours être compris comme une construction ou une fiction sociale. L'un des fondements de la méthodologie wébérienne, le postulat de la neutralité axiologique, semble ne laisser aucune marge de manœuvre: de la même façon qu'il paraît ne pas convenir de parler de l'origine surnaturelle du charisme et de ses qualités, il semble également interdit de différencier un charisme véritable d'un faux, un charisme authentique d'un charisme artificiel ou fabriqué. La sociologie semble avoir à accepter l'interprétation du charisme telle qu'elle est définie par les acteurs comme réalité sociale, et à rendre compte de ses conditions, fonctions et conséquences. Elle ne remettra en aucun cas le charisme en question, pas même par exemple «l'objectivité » de la revendication charismatique. Il s'agit ici d'une question de principe. Une telle remise en question serait, selon ce principe, aussi inadmissible et irréalisable que la proposition du défunt Wolfgang J. Mommsen, important historien et wébérologue, qui voulait qu'en aucune circonstance ne soient qualifiés de charismatiques des chefs indubitablement méchants, Hitler par exemple (1985).

Mais la distinction entre un charisme authentique et un charisme artificiel (ou entre un charisme fort et un charisme faible) doit-elle inévitablement reposer sur un jugement de valeur, en particulier sur un jugement de valeur de nature morale? Est-il interdit — et, pour la sociologie, logiquement impossible — d'étudier, à l'aide de ces distinctions ou d'autres du même genre, les qualités et les capacités tenues pour extraordinaires de certains individus? D'interroger si, et dans quelles mesures, celles-ci existent ou ont existé ? Les sociologues sont-ils ici soumis, comme dans l'étude de la religion ou de l'esthétique, à une abstention de tout jugement?

Il n'y a rien à objecter à une telle abstention de jugement à laquelle dérogent souvent de facto les études empiriques, et ce, même sous la plume 
de Max Weber. La justesse de normes logiques ou morales n'est pas nécessairement remise en question par des arguments dont la logique laisse à désirer ou par des actions amorales. Le fait que Weber affirme que le concept de charisme est utilisé d'une façon tout à fait neutre du point de vue des valeurs $(1995,321)$ ne l'empêche pas de constamment distinguer le charisme "authentique» d'autres formes de charisme. Face à une telle situation, le regard critique devrait se porter moins sur l'exigence de la neutralité en valeur que sur Weber lui-même.

Il semble cependant que cette distinction entre charisme authentique et charisme artificiel, particulièrement dans le contexte d'une société de masse et des médias, soit incontournable ${ }^{9}$. Voici donc l'occasion d'examiner ce que l'on entend (ou pas) par une telle distinction.

Ici comme ailleurs, on doit différencier une distinction normative d'une distinction objective, non normative (et cela surtout lorsque cette distinction tend à impliquer une évaluation, un jugement). Il s'agit de découvrir si, ou du moins à quel point, une chose qui est définie comme vraie par les acteurs sociaux (mais qui peut être évaluée de diverses manières par les sociologues) existe réellement. Au lieu d'analyser simplement les qualités et les compétences qualifiées de "charismatiques" par les acteurs sociaux et aussi, peut-être un peu précipitamment, par des sociologues comme Max Weber, le sociologue peut s'interroger sur l'existence et l'effet réel de telles qualités. De telles analyses pourront mener à la conclusion qu'il ne s'agit que de simples attributions, et donc de rien d'autre que de constructions ou de fictions sociales. Elles pourront également laisser conclure que le caractère extraordinaire attribué à ces capacités est réel. Un tel cas sera avéré lorsque le caractère extraordinaire sera indubitablement démontré et lorsque certains accomplissements, aussi bien au plan religieux ou moral que pratique, ne se laisseront expliquer que par ce caractère extraordinaire.

De par la nature de ses moyens, la sociologie ne peut affirmer ou rejeter l'origine divine d'une capacité extraordinaire. Elle peut (et pourra) cependant considérer une telle conviction comme un facteur souvent (mais pas toujours) important pour rendre compte de ce phénomène «d'abandon plein de foi » (gläubige Hingabe).

9. Gebhardt évoque (1993, 12 note 14) aussi une littérature récente et foisonnante sur la fabrication et l'influence du «charisme artificiel». 
12.

Dans sa démarche vers une "transparence causale", la sociologie peut se voir contrainte à prendre acte du fait que certaines grandes et importantes créations sociales ou sociopolitiques (y compris celles qui se révèlent des plus éphémères ou destructives) ne se laissent suffisamment expliquer que par un recours aux propriétés et aux capacités extraordinaires d'individus particuliers.

C'est ici que sont assignées la position et la signification du charisme, dans sa forme originaire ou authentique, dans le système théorique de la sociologie historique de Max Weber. Le charisme est pour lui, dans un sens purement empirique et neutre du point de vue des valeurs, la grande puissance "spécifiquement révolutionnaire" de l'histoire (1995, 323-324). Il entend par là l'histoire des religions (et des guerres) en général et l'histoire occidentale des ruptures et innovations politiques en particulier.

Weber considère toujours les prophètes comme des "porteurs de charisme ». Ce n'est pas par hasard qu'ils représentent le point de comparaison qu'il oppose aux revendications charismatiques de la "prophétie en chaire » (Professoren-Prophetie) de son époque. Dans les paroles de Jésus de Nazareth, "c'est écrit, je vous le dis ", il voit articulé de la façon la plus achevée le credo et la prétention à une domination charismatique. La "prophétie authentique» (genuine Prophetie) de l'Ancien Testament n'est pas seulement un objet de recherche particulier auquel se serait consacré Weber avec une intensité particulière et une sympathie manifeste; elle a pour Weber un statut exemplaire. Elle représente ce que recèlent le charisme authentique et la domination purement charismatique; le lieu où se fondent sa puissance révolutionnaire et son efficacité. De toute évidence, Weber considère que le charisme se manifeste ici dans une forme dont la pureté n'existe que dans le type idéal $(1995,326)$. Le fait que la revendication de ces prophètes à diriger soit fondée sur des motifs "purement religieux " caractérise, pour Weber, la pureté idéaltypique de cette forme de domination.

Pour Weber, les grands bouleversements historiques en général et les renouveaux religieux en particulier ne peuvent être déclenchés et mis en branle que "de l'intérieur, en provoquant une "metanoia" centrale dans l'état d'esprit des dominés" $(1972,657 ; 2005,127)$ grâce à des individus dotés de charisme. Si l'on suit cette hypothèse, on s'explique (au regard de sa très grande exigence envers le charisme véritablement religieux) pourquoi il se montrait si sceptique quant au destin de la religion dans un futur proche, du moins en Occident. Personne ne sait, nous dit-il en conclusion 
de L'éthique protestante, si s'accomplit "cette terrible évolution» vers une "pétrification mécanisée " et un monde organisé selon les besoins de ceux décrits par Nietzsche dans Ainsi parlait Zarathoustra comme les «derniers hommes ", ou si elle mènera à "une puissante renaissance de conceptions anciennes et d'idéaux du passé » ou encore à "des prophètes entièrement nouveaux» (2002, 302). Il clôt le testament critique de son époque, la conférence La science, profession et vocation, en proposant que "tous ceux, et ils sont nombreux, qui attendent aujourd'hui de nouveaux prophètes et sauveurs " soient renvoyés "au chant de veilleur édomite qui était chanté au temps de l'Exil et fut repris dans les oracles d'Isaïe » (Is 21,11-12): «le matin vient, mais c'est encore la nuit. Si vous voulez encore poser la question, revenez une autre fois» $(2005,59)$.

\section{3.}

Nonobstant ce que la sociologie peut accomplir par l'étude du charisme (et contrairement à une opinion très répandue), elle est, grâce à cet objet de recherche plus qu'à tout autre, confrontée à ses limites ${ }^{10}$. Toutefois, à l'opposé de ce que l'on pourrait penser et craindre, il ne s'agit pas d'une faiblesse de cette science humaine, mais plutôt d'un potentiel critique guère exploité. Critiquer signifie distinguer, différencier. La sociologie est critique lorsqu'elle confronte une réalité sociétale donnée, non pas à une évaluation ou à un jugement (moral ou politique), mais plutôt à des différenciations qui lui sont propres. Ce n'est qu'ainsi qu'une explication sociologique est possible: une connaissance et une perception adéquate d'une réalité sociétale donnée n'apparaissent qu'à l'aune d'une telle différenciation. Le sens conféré au charisme et la rhétorique du charisme sont en ce sens des objets particulièrement importants et féconds d'une sociologie soucieuse de distinguer et de critiquer.

Une sociologie qui se comprend et procède de la sorte se révèle également critique dans un second sens «réflexif ». Cette science est encline, comme il a été souligné précédemment, à dépasser ses propres frontières et à s'approprier l'explication de l'ensemble de la réalité humaine. Cette prétention à l'hégémonie a porté préjudice au prestige de la sociologie. Ceci laisse à penser en soi, mais il semble plus important encore que la sociologie soit ainsi restée en deçà de ses possibilités. La sociologie est en effet confrontée,

10. Le «charisme» n'est donc pas une "catégorie résiduelle", mais plutôt un concept limitrophe de la sociologie. 
certes à sa limite mais pas uniquement à la marge, à des réalités dont la portée sociale est grande sans que celles-ci ne se laissent expliquer de façon exhaustive avec ses outils. Ces réalités ne sont pas "conditionnées socialement ", mais elles peuvent influencer, voire modifier de façon radicale les conditions sociales. Ce changement se produit grâce à l'intervention d'individus extraordinaires et charismatiques qui réforment de fond en comble des structures sociales ou mentales pour créer du nouveau, et dont la force peut déclencher une impulsion destructrice inouï.

Pourtant, tout cela se déroule aussi continuellement d'une façon à peine perceptible, bien que fondamentale, parce que les acteurs sociaux ne sont jamais uniquement sociaux, mais toujours également singuliers, uniques. Georg Simmel a formulé cette importante idée dans le second a priori de sa célèbre digression Comment la société est-elle possible?: "pour l'individu [...] sa manière d'être socialisé est déterminée ou codéterminée par sa manière de ne pas être socialisé » $(1999,71)$. Pour Simmel, jusqu'à ce jour de loin le plus important théoricien de l'individualité et de l'individualisation en sociologie, l'individualité qui s'élude de toute socialisation et qui, en même temps, participe à cette socialisation devint un thème si important qu'à la fin il détourna toute son énergie de la sociologie au profit d'une «métaphysique de l'individualité».

\section{4.}

Kierkegaard avait parlé de la double individualité: d'une part, "au sens de la distinction ou du talent spécial "; d'autre part, "au sens où tout homme sans exception peut et doit l'être» (1971, 93). Il semble paradoxal, mais digne de considération, que ces deux (différentes, mais liées l'une à l'autre) formes d'existence de l'individu aient façonné l'horizon de pensée de Georg Simmel et de Max Weber. Ces deux classiques de la sociologie ont chacun thématisé avec insistance une de ces formes et en ont fait la pierre de touche de la connaissance sociologique. Lorsque la sociologie appréhende et prend au sérieux l'individualité, dans ses formes quotidiennes ou extraquotidiennes, elle peut être certaine d'aller jusqu'à la frontière de ce que lui rendent possible ses moyens. Si cette certitude, réflexive et justifiée, ne va pas de soi, elle s'avère très satisfaisante et productive d'un point de vue intellectuel. Il ne peut être qu'à l'avantage de la précision des analyses sociologiques et de la justesse de leurs descriptions du réel qu'elles considèrent le général du point de vue de ce qui est exclu de ce général, mais qui n'en est toutefois pas séparé. 
Lorsque l'on se rapproche de cette problématique du point de vue de Kierkegaard, comme ce fut le cas ici, on est enclin à penser qu'il en va aussi chez ces grands sociologues d'une influence souterraine ou d'une réinterprétation d'idées religieuses et théologiques, peut-être même d'idées dont on peut retracer l'origine chez Kierkegaard. Cela ne constituerait toutefois pas, en soi, une réfutation d'une telle problématique et de son approche.

\section{Références}

Breuer, S. (1993), "Das Charisma der Vernunft », dans W. GebHard, A. Zingerle, et M. N. EberTZ, dir., Charisma. Theorie, Religion, Politik, Berlin/New York, Gruyter, p. 159-184.

(1993), «Einleitung. Grundlinien der Entwicklung des Charismakonzeptes in den Sozialwissenschaften", dans W. GebHard, A Zingerle, et M. N. Ebertz, dir., Charisma. Theorie, Religion, Politik, Berlin/New York, Gruyter, p. 1-12.

GEBHARDT, W. (1994), Charisma als Lebensform. Zur Soziologie des alternativen Lebens, Berlin, Dietrich Reimer Verlag.

Gebhardt, W., A. Zingerle, et M. N. Ebertz, dir., (1993), Charisma. Theorie, Religion, Politik, Berlin/New York, Gruyter.

KALINOWSKI, I. (2005), «La voix de Max Weber et le charisme professoral », dans La science, profession et vocation. Suivi de Leçons wébériennes sur la science et la propagande, Marseille, Agone.

KierkegaArd, S. (1971) [1849], CEuvres complètes, t. 16: Point de vue explicatif de mon CEuvre d'écrivain. Deux petits traités éthico-religieux. La maladie à la mort. Six discours, Paris, De l'Orante.

l'Orante.

(1979) [1846], CEuvres complètes, t. 7: Trois discours sur des circonstances supposées. Quatre articles de Fodrelandet. Un compte rendu littéraire, Paris, De l'Orante.

Lamartine, A. de (1847), Histoire des Girondins, 33/3, Paris, Furne.

LePsIUS, M. R. (1981), «Charismatic Leadership. Max Weber's Model and its Applicability to the Rule of Hitler ", dans C. F. Graumann et S. MoscovicI, dir., Conceptions of Leadership, New York, Springer, p. 53-66. 
LuKÁCs, G. (2006) [1954], La destruction de la raison. Nietzsche, Paris, Delga.

Mommsen, W. (1985) [1974], Max Weber et la politique allemande, 18901920, trad. Par J.-R. Amsler et al., Paris, PUF.

RIEFF, P. (2008), Charisma. The Gift of Grace, and How it Has Been Taken Away from Us, New York, Vintage Books.

Roth, G. (1987), Politische Herrschaft und persönliche Freiheit. Heidelberger Max Weber Vorlesungen 1983, Francfort, Suhrkamp.

SCHLUCHTER, W. (1991), «Umbildung des Charismas. Überlegungen zur Herrschaftssoziologie ", dans Religion und Lebensfübrung, t. 2, Francfort, Suhrkamp, p. 535-554.

Simmel, G. (1999) [1908], Sociologie. Études sur les formes de la socialisation, Paris, PUF.

Weber, M. $\left(1972^{5}\right)$, Wirtschaft und Gesellschaft, Tübingen, Mohr.

- (1995) [1921], Économie et société, trad. sous la direction de Chavy et de Dampierre, Paris, Plon.

(1996) [1915], "Introduction », dans Sociologie des religions, trad. par J.-P. Grossein, Gallimard, Paris, p. 331-378.

(2002) [2000, 1904], L'éthique protestante et l'esprit du capitalisme, trad. par I. Kalinowski, Paris, Flammarion.

- (2002), Schriften 1894-1922, Stuttgart, Kröner.

(2005) [1917], La science, profession et vocation. Suivi de Leçons wébériennes sur la science et la propagande, trad. par I. Kalinowski, Marseille, Agone.

WeIss, J. (1993), Vernunft und Vernichtung. Zur Philosophie und Soziologie der Moderne, Opladen, Westdeutscher Verlag.

(1998a) [1986], Weber and the Marxist World. Max Weber Classic Monographs, t. 6, London/New York, Routledge.

(1998b), Handeln und handeln lassen. Über Stellvertretung, Opladen/Wiesbaden, Westdeutscher Verlag. 


\title{
Résumé
}

Le présent essai cherche à observer et à comprendre pourquoi la sociologie s'accommode si difficilement des exceptions dont la portée est générale, exceptions qui se caractérisent par une «distinction ou [... un] talent spécial ». Pour ce faire, il retrace d'abord le traitement du charisme dans la discipline sociologique à partir de la réception des écrits de Max Weber sur ce concept. Il plaide ensuite pour un retour à la position originale de Weber, pour qui le charisme ne saurait être réduit à une "catégorie résiduelle », mais représente bien plutôt un concept limitrophe et fécond de la sociologie.

\begin{abstract}
This essay aims at observing and understanding why it is difficult to consider from a sociological perspective some exceptions that have widespread consequences - exceptions that are characterized by a «distinction or [... a $]$ special talent». Through the reception of Max Weber's work, it first reconstructs how the concept of charisma has been treated in sociology. Then, it suggests returning to Weber's original position, for whom charisma cannot be confined to a "residual category», but rather be considered as a border yet fecund concept of sociology.
\end{abstract}

